\section{The Products of Hyperreal Series and the Limitations of Cauchy Products}

Jonathan Bartlett

DOI: 10.33014/issn.2640-5652.3.2.bartlett.1

\section{Abstract}

Cauchy products are used to take the products of convergent series. Here, we show the limitations of this approach in divergent series. Alternative approaches and formulas for divergent series are suggested, as well as their benefits and drawbacks.

Bartlett, Gaastra, and Nemati (2020) set out to define a new way of assigning values to divergent series using hyperreal numbers, which we will refer to as the BGN method. This method operates using a small number of principles to allow value assignment for a divergent series:

1. Instead of summing to the ambiguous $\infty$, summations are done to a specific standard candle of hyperreal infinity, labeled $\omega$.

2. Because summations are done to a specific infinity, then making sure that the number of positions are maintained during manipulations is important.

3. When these rules are kept, divergent series summation can be done just like partial sums up to a value of $k$, where $k=\omega$.

Because of principle 2, $1+2+3+\ldots$ refers to a different value than $1+0+2+0+3+0+\ldots$, because the latter only has non-zero numbers in half of the positions as the former. To get both of these series to sum to the same value, the former series would sum from 1 to $\omega$, while the later series would have to sum from 1 to $2 \omega$. Because of principle 3 , rearrangements of the series do not affect the value of the series. Additionally, because these results operate identically to partial sums, standard summation formulas and discrete integrals can be used to simplify results.

As an example, the series

$$
A=\sum_{i=1}^{\omega} i
$$

Figure 1: Cauchy Multiplication of Six Terms

\begin{tabular}{c|c|c|c|c|c|c} 
& $a_{1}$ & $a_{2}$ & $a_{3}$ & $a_{4}$ & $a_{5}$ & $a_{6}$ \\
\hline$b_{1}$ & $a_{1} b_{1}$ & $a_{2} b_{1}$ & $a_{3} b_{1}$ & $a_{4} b_{1}$ & $a_{5} b_{1}$ & $a_{6} b_{1}$ \\
\cline { 2 - 7 }$b_{2}$ & $a_{1} b_{2}$ & $a_{2} b_{2}$ & $a_{3} b_{2}$ & $a_{4} b_{2}$ & $a_{5} b_{2}$ & $a_{6} b_{2}$ \\
$n_{3}$ & $a_{1} b_{3}$ & $a_{2} b_{3}$ & $a_{3} b_{3}$ & $a_{4} b_{3}$ & $a_{5} b_{3}$ & $a_{6} b_{3}$ \\
\cline { 2 - 6 }$b_{4}$ & $a_{1} b_{4}$ & $a_{2} b_{4}$ & $a_{3} b_{4}$ & $a_{4} b_{4}$ & $a_{5} b_{4}$ & $a_{6} b_{4}$ \\
\cline { 2 - 6 }$b_{5}$ & $a_{1} b_{5}$ & $a_{2} b_{5}$ & $a_{3} b_{5}$ & $a_{4} b_{5}$ & $a_{5} b_{5}$ & $a_{6} b_{5}$ \\
\cline { 2 - 6 }$b_{6}$ & $a_{1} b_{6}$ & $a_{2} b_{6}$ & $a_{3} b_{6}$ & $a_{4} b_{6}$ & $a_{5} b_{6}$ & $a_{6} b_{6}$
\end{tabular}

can be given a value using the standard arithmetic series formula, yielding a value of $\frac{\omega^{2}}{2}-\frac{\omega}{2}$. This can be rounded to the primary part of the result, $\frac{\omega^{2}}{2}$.

Essentially, using hyperreals allows one to treat divergent series as if they were finite series. The point of this article is to point out that, while this makes divergent series work as if they were finite series, it does not make divergent series work as if they were convergent series. This is an important distinction that is easy to miss.

\section{The Cauchy Product}

To highlight the importance of this distinction, we will focus on the Cauchy product. The Cauchy product for two series $A$ and $B$ is

$$
A \cdot B=\sum_{i=1}^{\omega}\left(\sum_{j=1}^{i} a_{j} b_{i-j+1}\right) .
$$

For convergent series, the Cauchy product is equivalent to the product of $A$ and $B$. However, even with hyperreals, the Cauchy product is not equivalent to the product of $A$ and $B$ if $A$ and $B$ are divergent.

To understand why, it is important to recognize the shape of the Cauchy product for finite series. Imagine that $A$ and $B$ are finite series with $k$ elements. In that case, it is easy to recognize that the sum does not work.

Let us imagine two series with six elements. The Cauchy product for such a series would be

$$
A \cdot B=\sum_{i=1}^{6}\left(\sum_{j=1}^{i} a_{j} b_{i-j+1}\right) .
$$

Figure 1 shows what this would look like. Each stripe of the figure represents one iteration through the outermost summation. Notice, however, that there are no stripes past the center stripe. In other words, all of the values past the center stripe are not considered in the final summation.

This is obviously problematic for finite sums. Why is it nonproblematic for convergent series? 
Figure 2: The Rectangular Product of $A$ and $B$

\begin{tabular}{c|ccc|c|c|c|} 
& $a_{1}$ & $a_{2}$ & \multicolumn{1}{c}{$a_{3}$} & $a_{4}$ & $a_{5}$ & $a_{6}$ \\
\hline$b_{1}$ & $a_{1} b_{1}$ & $a_{2} b_{1}$ & $a_{3} b_{1}$ & $a_{4} b_{1}$ & $a_{5} b_{1}$ & $a_{6} b_{1}$ \\
\cline { 2 - 7 }$b_{2}$ & $a_{1} b_{2}$ & $a_{2} b_{2}$ & $a_{3} b_{2}$ & $a_{4} b_{2}$ & $a_{5} b_{2}$ & $a_{6} b_{2}$ \\
$b_{3}$ & $a_{1} b_{3}$ & $a_{2} b_{3}$ & $a_{3} b_{3}$ & $a_{4} b_{3}$ & $a_{5} b_{3}$ & $a_{6} b_{3}$ \\
\cline { 2 - 7 }$b_{4}$ & $a_{1} b_{4}$ & $a_{2} b_{4}$ & $a_{3} b_{4}$ & $a_{4} b_{4}$ & $a_{5} b_{4}$ & $a_{6} b_{4}$ \\
$b_{5}$ & $a_{1} b_{5}$ & $a_{2} b_{5}$ & $a_{3} b_{5}$ & $a_{4} b_{5}$ & $a_{5} b_{5}$ & $a_{6} b_{5}$ \\
$b_{6}$ & $a_{1} b_{6}$ & $a_{2} b_{6}$ & $a_{3} b_{6}$ & $a_{4} b_{6}$ & $a_{5} b_{6}$ & $a_{6} b_{6}$ \\
\cline { 2 - 7 } & & & & & &
\end{tabular}

For a convergent series, as the index approaches infinity, the value of the the term approaches zero. If you look at Figure 1, it is evident that the terms that are being ignored, if it is a convergent series, have at least one multiplicand of the term at a near-zero value. Therefore, in a convergent series, all of the terms being ignored are negligible.

However, that is not true of a finite series. When performing hyperreal summations, the values and manipulations are similar to finite series, not necessarily similar to convergent series.

\section{Non-Cauchy Products}

An advantage of Cauchy products is that it defines a way of understanding the behavior of a series in terms of a non-trivial manipulation of the previous series. It is obvious that we could represent the multiplication of hyperreal series $A$ and $B$ as

$$
A \cdot B=\left(\sum_{i=1}^{\omega} a_{i}\right) \cdot\left(\sum_{i=1}^{\omega} b_{i}\right)
$$

but that gives little additional information.

Another way of considering the multiplication is to take each element of $A$ and multiply it by each element of $B$. This can be expressed as

$$
A \cdot B=\sum_{i=1}^{\omega}\left(\sum_{j=1}^{\omega} a_{i} b_{j}\right) .
$$

This, however, is not as useful as it could be. Since it basically goes through every element of $B$ before considering even the second element of $A$, partial sums will not provide much information about the behavior of the product as a whole.

A more informative formula can be found by tracing paths as outlined in Figure 2, which we will call the rectangular method. This is in contrast with the Cauchy multiplication method, which is triangular, removing half of the terms from consideration. In a rectangular product, at each index of the outer sum, all of the indices in both $A$ and $B$ up to that index are considered.
Figure $3:(1+1+1+\ldots) \cdot(1+-1+0+0+0+\ldots)$

\begin{tabular}{c|c|c|c|c|c|c} 
& 1 & 1 & 1 & 1 & 1 & 1 \\
\hline 1 & 1 & 1 & 1 & 1 & 1 & 1 \\
\cline { 2 - 6 }-1 & -1 & -1 & -1 & -1 & -1 & -1 \\
0 & 0 & 0 & 0 & 0 & 0 & 0 \\
0 & 0 & 0 & 0 & 0 & 0 & 0 \\
0 & 0 & 0 & 0 & 0 & 0 & 0 \\
0 & 0 & 0 & 0 & 0 & 0 & 0
\end{tabular}

This method can be represented by the formula

$$
A \cdot B=\sum_{i=1}^{\omega}\left(\left(\sum_{j=1}^{i} a_{i} b_{j}\right)+\left(\sum_{k=1}^{i-1} a_{k} b_{i}\right)\right) .
$$

Because this formula incorporates every term of the series $A$ and $B$, then the result will be equal to the naive multiplication in (4). Additionally, to the extent that the beginning of the series are representative of the series as a whole, this will allow the behavior of partial sums of the series to be used as approximations of the behavior of the series as a whole in certain circumstances. It also has the same number of elements as both the multiplicand series, so it can easily be used in operations with other series having the same number of infinite terms, which can be helpful in the context of the BGN series summation method.

\section{An Example}

The multiplication that first sparked this consideration is the product of the series $1+1+1+\ldots$ multiplied by the series $1+-1+0+0+0+\ldots$ (with zeroes continuing). When put in the form specified by (1), the former sequence is equivalent to $\omega$. The latter sequence is obviously 0 .

Although $\omega \cdot 0=0$, the Cauchy product of these two series actually turns out to be 1 . The reason for this can be seen in Figure 3. As can be seen, everything is included in this product except one term: -1 . Therefore, since the entire product is 0 , the product without the final -1 term will be 1 .

This Cauchy product has this shape no matter how many terms (even an infinite number of terms), and therefore will always have the result of 1 . However, in both the finite case and the infinite case, the actual product of the two series is zero.

\section{Additional Considerations}

The considerations given here should demonstrate why Cauchy products can be useful for convergent series while not being 
useful at all for multiplying two divergent series. Additionally, it should be evident that, even if the formula for the Cauchy product of a divergent series converges, it does not mean that the Cauchy product represents in any significant way the true value of the product of the two series. It simply means that, when half of the terms are not considered, the result is convergent. That does not yield a significant amount of confidence in such a result.

\section{Conclusions and Clarifications}

In (Bartlett, Gaastra, and Nemati, 2020), Section 11.2 hedged on the consideration of series rearrangement, suggesting that we could not rule out that rearranging series might cause the series to differ by an infinitesimal. However, the problem was that we were considering the results of Cauchy Products, which, here, we have shown are not representative of true products of divergent series.

As already noted, divergent series can, using hyperreals, be evaluated in the same way as if they were finite series. However, even though they can be treated similar to finite series, that is not the same as saying that they can be treated as convergent series.

Bartlett, J, L Gaastra, and D Nemati (2020). "Hyperreal Numbers for Infinite Divergent Series". In: Communications of the Blyth Institute 2.1, pp. 7-15.

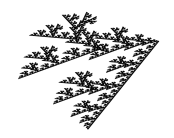

\section{Following the Science}

Tom and Elizabeth Siewert

DOI: 10.33014/issn.2640-5652.3.2.siewert.1

More and more frequently we are hearing the words "follow the science" spoken by those who believe that they are right and are frustrated by those who disagree with them. It sounds good: we should avoid illogical or emotional responses and just focus on what is proven to be correct. Of course, no one wants to be wrong, and so we tend to just go along with the opinion of experts in the field. We don't want to waste time in rehashing old arguments that have been proven to be incorrect. Let's just move on.
But what if this phrase is used to stifle questions that don't fit a socially or politically accepted idea, especially if the questions are targeted at some weakly supported foundational concepts? If you don't want to permit debate on a topic that you might not decisively win, you might just tend to bluster your way out through intimidation. Stating "Follow the Science" implies that the opposing view is unscientific, and so should be immediately discounted and dropped from consideration. How could anyone possibly support such a weak idea?

Are there any specific examples in which the "Follow the Science" slogan has been used to support an increasingly weak argument and deflect a question that can't be clearly countered? We think that the complexity of cells is one such area. It is framed in the struggles between the two camps: Evolution versus Intelligent Design.

Take, for example, the article "The Flaws in Intelligent Design" (Collinsworth, 2006). In explaining what is wrong with Intelligent Design, the author first characterizes ID proponents as those who want to "challenge evolution and promote fundamentalist-friendly 'science' in public education and public discourse" implying that ID proponents are not really scientists. It then goes on to state:

\begin{abstract}
"Regarding ID's specific claims, scientists object that the concept of "irreducible complexity" relies upon a mischaracterization of biological mutation as a relatively linear process involving only the addition of more and more "parts," rather than a dynamic process that can also reshape, rearrange, or fundamentally alter existing elements and features. Systems that must be fully formed to serve their current function could have developed from earlier forms that served a different function, or could be significantly reorganized versions of an earlier form that served the same function.

Mathematicians are similarly critical of ID's mathematical arguments against evolution, which rely on an excess of subjective calculations, manipulation of numbers, and misrepresentations of evolutionary models."
\end{abstract}

The narrative in the first paragraph about dynamic reshaping and rearranging of existing elements would be more compelling if it included references to data, observations, or at least models of such modified biological forms, rather than just a shallow statement that it "could have developed". The best long-term data on real-life mutations is the 50,000 generation study of E. coli by the Richard Lenski lab at Michigan State University (Lenski, 2021). The data shows only a "relatively linear" and minor history of changes, rather than dynamic rearranging 